\title{
Narrativas en red: dominación o agencia en discursos de Twitter y YouTube frente al fenómeno del feminicidio en México
}

\author{
Sección: Dossier \\ Recibido: 30/09/2021 \\ Aceptado: 15/11/2021
}

\author{
Networked Narratives: Domination or Agency in Twitter and \\ YouTube Discourses in the Face of the Femicide Phenomenon in \\ Mexico
}

\author{
Carolina Hernández Garza \\ Tecnológico de Monterrey, México \\ https://orcid.org/0000-0001-8146-9828 \\ cahega22@gmail.com
}

\begin{abstract}
Resumen
El presente artículo propone analizar la generación de contenido discursivo en Twitter y YouTube frente al más reciente caso mediático de feminicidio en el país, el feminicidio de Montserrat Bendimes, con el fin de explorar en ambas plataformas las narrativas en red que allí se generan. Lo anterior, mediante un proceso de scrapping de tuits encontrados en el hashtag \#justiciaparamonse en Twitter y de comentarios generados en dos videos sobre el caso observados en la plataforma YouTube, lo cual fue analizado a través de la aplicación de código abierto en web para análisis de texto Voyant Tools. Recurriendo a una visión situada de la teoría del tecnofeminismo de Judy Wajcman, se busca explicar si las narrativas encontradas en cada una de las plataformas analizadas permiten observar dinámicas de agencia feminista que brinden resistencia a la comunicación convencional de los medios tradicionales en el país frente al fenómeno a estudiar, o bien, si responden a dominaciones discursivas basadas en las violencias de género que el activismo feminista ha buscado destituir.
\end{abstract}

Palabras clave: Narrativas en red, Twitter, YouTube, tecnofeminismo, feminicidio.

\section{Introducción}

En México, la violencia de género, cuyo último extremo visible es el feminicidio, representa una problemática creciente que paulatinamente colectivas feministas, académicas y mujeres han buscado posicionar en la esfera de la discusión pública después de estar históricamente mitificada en la media convencional. Considerado como uno de los epicentros de violencia de género, en los primeros cinco meses del 2021 se reportaron en el país 423

\begin{abstract}
This article proposes analyzing the generation of discursive content on Twitter and YouTube in the face of the most recent media case of femicide in the country, the femicide of Montserrat Bendimes, to explore the networked narratives generated on both platforms. It is done by scrapping both tweets found in the hashtag \#justiciaparamonse on Twitter and comments generated in two videos about the case found on YouTube. This analysis uses Voyant Tools for digital texts. Using a decolonial vision of Judy Wajcman's theory of techno-feminism, the article seeks to explain whether the narratives in these platforms allow the observation of feminist agency dynamics that provide resistance to the country's conventional communication in traditional media of the phenomenon under study. Otherwise, if they respond to discursive dominations based on gender violence that feminist activism has sought to transmute.
\end{abstract}

Keywords: Networked narratives, Twitter, YouTube, techno-feminism, femicide. 
víctimas de feminicidio, un $7.1 \%$ de incremento con respecto al mismo periodo del año anterior (Guillén, 2021). Además del incremento de este tipo de asesinatos por violencia de género, existe un nivel de impunidad del $51.4 \%$, donde solo un $26.4 \%$ de las muertes violentas de mujeres son indagadas como feminicidios (Forbes Staff, 2020). A esto, se le añade la manera en la que, tradicionalmente, la prensa y los medios convencionales de comunicación en México han reportado los casos de feminicidios ocurridos en territorio nacional, considerando la cobertura periodística del feminicidio como mitificada y desvinculada de la violencia estructural (Hernández Montoya, 2020).

Según McNeill (2006), este tipo de crímenes suele mitificarse dentro de la redacción y los encuadres de la comunicación periodística, al relacionar los hechos con tragedias amorosas, pleitos conyugales o condiciones y relaciones sociales específicas. Siguiendo el estudio realizado por Hernández Montoya, en México "la redacción de dichas notas refuerza la ideología machista que justifica y minimiza las agresiones contra las mujeres, desvinculándola de la violencia estructural y llevándola al plano de lo privado" (2020, párr. 4). Este encuadre otorgado en su mayoría en los medios convencionales, ha impactado en la percepción social que se tiene frente al fenómeno de los feminicidios en el país (Tiscareño y MirandaVillanueva, 2020), imposibilitando abordarlo como una problemática estructural y sistémica a partir de una perspectiva de género, así como de suscitar debates en la esfera pública que pongan en evidencia la necesidad de generar políticas robustas para la investigación, reconocimiento, prevención y vinculación a proceso para la reparación de daños de este tipo de delitos contra las mujeres.

En este contexto, podría considerarse que la prensa y los medios tradicionales "difícilmente rinden cuentas" frente al fenómeno mencionado, y producen un vacío que a su vez resulta en una exigencia de reconocimiento de que "la información y la comunicación son bienes públicos sobre los cuales la ciudadanía puede ejercer derechos” (Sandoval, 2010, p. 60). Sin embargo, en una dominación aún visible de los medios que tradicionalmente funcionan como difusores de las discusiones públicas, es que surge la necesidad de generar y estudiar procesos comunicacionales frente a las coyunturas de feminicidios en el país, a través de lo que Zizi Papacharissi (2014) denomina como electronic elsewheres o plataformas digitales sociotécnicas como lo son Twitter y YouTube, donde pueda existir una aproximación distinta a los códigos hegemónicos que han operado hasta ahora, y que funcionen como arenas capaces de dar apertura a contranarrativas, exigencias y demandas de públicos alternos.

No obstante, una concepción meramente optimista frente al uso de las plataformas digitales como espacios para la demanda política feminista cae en un importante riesgo de ser esencialista y utópica, en tanto que tiende a olvidar el carácter constitutivo que el género ha tenido sobre la tecnología y sobre el ciberespacio, y donde se hacen presentes nuevas formas de violencia de género que no pueden ser obviadas ni eludidas. Por ello, el presente artículo retoma como punto de partida la teoría del tecnofeminismo de Judy Wajcman (2006), donde se reconoce un punto intermedio entre visiones tecnófobas y tecnofílicas del feminismo con respecto a la tecnología, situándola en un contexto decolonial donde es posible observar nociones particulares de agencia y, a la par, de dominación en las narrativas suscitadas en plataformas digitales.

Considerando el concepto de 'narrativas en red' como discursos coproducidos por públicos integrados en plataformas digitales (Page et al., 2013, p. 194), el presente artículo busca analizar la generación de narrativas en los espacios de Twitter y YouTube frente al más 
reciente caso mediático coyuntural en el país, el feminicidio de Montserrat Bendimes. Esto, con el fin de explorar en ambas plataformas las narrativas en red que allí se generan y en qué medida estas responden ya sea a dominaciones discursivas basadas en una visión patriarcal del fenómeno, o bien, a dinámicas de agencia feminista que, a través de contranarrativas, brinden resistencia a la comunicación convencional encontrada en los medios tradicionales en el país.

\section{Marco Teórico}

\section{Tecnofeminismo: aproximación teórica como punto de partida}

Con la pregunta inicial — “iexiste alguna alternativa más allá de dos opciones limitadas: rechazar de plano las tecnologías existentes o adoptar sin sentido crítico el cambio tecnológico?" (Wajcman, 2006, p.15), Judy Wajcman se ha vuelto un referente en la academia feminista desde que en el 2006 publicó un libro homónimo que presentaba los argumentos del tecnofeminismo como una nueva propuesta de relación entre el feminismo y la tecnología. El tecnofeminismo resulta de una hibridación entre las concepciones tecnofílicas del ciberfeminismo de los años noventa y las visiones tecnófobas de la década de los ochenta, en un intento por escapar de los esencialismos en los que incurrían estas perspectivas contrarias entre sí.

En primera instancia, el tecnofeminismo de Wajcman (2006, p. 16) reconoce a la tecnología como un producto socio-técnico que se conforma a partir de las relaciones sociales que lo producen y lo utilizan. Por ello, distingue que la tecnología no es neutral "sino que su creación y desarrollo responde a las estructuras sociales en las cuales surge" (Romero, 2014, p. 166), y donde los artefactos y redes tecnológicas deben ser concebidas como objetos culturales que cobran significado en un marco social. Al situar el carácter socio-técnico de la tecnología, y bajo la premisa de que la academia feminista ha reconocido ampliamente que el género está construido socialmente, Wajcman (2006) introduce una concepción "mutuamente conformadora entre género y tecnología, en la que la tecnología es al mismo tiempo fuente y consecuencia de las relaciones de género" (p. 17).

Por un lado, Wajcman (2006) cuestiona la perspectiva tecnófoba y de resistencia al uso y apropiación de la tecnología para prácticas feministas: "Inherente al planteamiento feminista radical es la concepción de la tecnociencia como intrínsecamente patriarcal" (p. 37), y aunque la autora no rechaza esta idea, puesto que reconoce que la tecnología, el Internet y las redes sociales están construidas sobre una cultura masculina que revela continuidades de poder y exclusión (p. 86), sí pone en cuestionamiento la tendencia esencialista que las plantea como herramientas inamovibles de dominación masculina y que "sitúa a las mujeres como víctimas uniformes de la tecnociencia patriarcal" (p. 40). Por ello, aunque reconoce que las concepciones sociales históricamente atribuidas al género han moldeado la construcción per se de aparatos y plataformas tecnológicas, así como las dinámicas embebidas en ellas, sostiene a su vez que el carácter sociotécnico de las mismas no las mantiene estáticas, sino que pueden ser replanteadas y reconformadas en tanto los usos sociales que se les otorguen.

No obstante, Wajcman (2006, p. 53) también ofrece una crítica al ciberfeminismo liberal por considerarlo utópico y altamente optimista respecto a la naturaleza de las tecnologías digitales y sus consecuencias para las mujeres. La propuesta del ciberfeminismo surgió en la década de los noventa frente a la esperanza depositada en el ciberespacio y la realidad virtual como nuevas arenas para socavar viejas relaciones sociales, así como para la 
liberación de los roles de género convencionales donde las mujeres pudieran aplicar sus propios fines sociales y políticos (p. 12). Esta visión pintaba a las nuevas tecnologías en red como excesivamente abiertas y maleables, lo que para Wajcman obviaba la necesidad de una tecnopolítica feminista que contrarrestara la utopía de la Web como zona de libertad sin límites para las mujeres y sus agendas particulares.

$\mathrm{La}$ autora del tecnofeminismo observa en el ciberfeminismo un riesgo esencialista de olvidar el carácter constitutivo que el género ha tenido sobre la tecnología y sobre el ciberespacio, y donde se hacen presentes nuevas formas de violencia de género que no pueden ser olvidadas ni eludidas por una corriente ultra optimista, como por ejemplo el auge de páginas web dedicadas a la pornografía, el acoso sexual digital, las redes de pedofilia y los ataques digitales a los que están expuestas las mujeres en mayor medida. Sin embargo, del ciberfeminismo recupera la propuesta de subjetividad y agencia que, a diferencia del feminismo radical, les otorga a las mujeres la capacidad de renegociar relaciones de poder entre los géneros dentro de los medios tecnológicos y plataformas digitales (Wajcman, 2006, p. $18)$.

Por ende, la teoría del tecnofeminismo resulta en un punto intermedio entre las dos posturas mencionadas con anterioridad, pues concuerda en el hecho de que la tecnología está conformada a partir de concepciones sociales de género donde se reproducen dinámicas de poder patriarcales, pero reconoce en ella un carácter sociotécnico que permite deconstruir la división diseñador/usuario de artefactos y plataformas tecnológicas (Wajcman, 2006, p. 74). De esta manera, al considerar a la tecnología y los espacios digitales como productos sociotécnicos, ve en ellos a la vez una capacidad emancipadora donde puede surgir la agencia humana que recupere al sujeto femenino dentro de la tecnología e identifique las distintas formas en que las mujeres resisten activamente a las construcciones convencionales de pasividad en estos espacios a la par que los subvierten (p. 169).

\section{Situando la agencia y el conocimiento tecnofeminista en un contexto decolonial}

La concepción tecnofeminista de las redes socio-técnicas de Wajcman (2006, p. 192) reconoce las diferentes formas en que las mujeres viven y experimentan la tecnociencia dependiendo de su emplazamiento. Sin embargo, es importante observar que las aproximaciones que éstas realizan se mantienen infundadas en una teorización y práctica occidental que no puede ser ignorada, a fin de reducir un esencialismo que deje atrás concepciones no-occidentales de personalidad, resistencia y agencia.

En este sentido, la teoría de la interseccionalidad permite comprender sistemas de poder que se cruzan: los sistemas de privilegios, por un lado, y sistemas de opresión, por otro, que determinan las experiencias particulares de una persona o grupo específico de personas. Este entendimiento brinda entonces la posibilidad de reconocer que existen distintas categorías de diferencia social, más allá del género -la cual ha sido únicamente analizada hasta ahora-, así como de observar situaciones en que personas o grupos enteros caen en dinámicas de olvido porque son doble o triplemente marginadas frente a otras condiciones como lo son la raza, la nacionalidad o la clase (D'Ignazio y Klein, 2020, p. 10).

Ante ello, autoras latinoamericanas como Natansohn y Paz (2018) y Reyes-Rivas (2017) comienzan por cuestionar los retos técnicos y de acceso al hablar de un ciberespacio en 
y desde América Latina. En palabras de Reyes-Rivas (2017), "a pesar de la supuesta transnacionalidad que se entiende como propia de los usos de las TIC (ciberespacio), ciertamente hay condiciones materiales dentro del subcontinente latinoamericano que no pueden ser obviadas a favor del acceso" (p. 156). De igual forma, Natansohn y Paz (2018) observan que las mujeres de las áreas pobres urbanas de países latinoamericanos tienen un 50\% menos oportunidad de acceso a Internet que los hombres de la misma región, y, por ende, menos posibilidad de utilizar plataformas digitales para su crecimiento económico y político, para buscar empleo y para participar de debates online. En este dato pueden encontrarse tres distintas categorías que atraviesan y limitan el acceso y participación tecnológica de las mujeres: género, clase y contexto geográfico, las cuales son reconocidas por la presente investigación para evitar caer en una concepción totalizadora y sobre-optimista del uso de las plataformas digitales para el activismo feminista.

Aún en este panorama que reconoce la interseccionalidad que afecta de manera particular a las mujeres en un contexto no-occidental, las autoras ya mencionadas encuentran en la tecnología — basadas en la concepción sociotécnica que propone Judy Wajcman (2006) - un escenario para la apropiación y la acción política feminista a partir de una noción decolonial que interpreta "los usos, adaptaciones y apropiaciones de símbolos, prácticas, objetos, estructuras y aparatos que generan la hibridez y la heterogeneidad sociotécnica" (Natansohn y Paz, 2018, párr. 15) en su contexto particular.

En este sentido, las políticas del reconocimiento cultural y decolonial "no se contraponen a las visiones feministas, sino que localizan las estrategias de lucha en contextos particulares" (Hernández Castillo, 2003, p. 33). Por ello, es posible reconocer y alentar a que, incluso bajo estructuras de dominación interseccionales y contextos estructurales que les son hostiles (Montanaro, 2017), las mujeres en contextos decoloniales puedan mantener y generar dinámicas de agencia y protesta social a través de la tecnología y las plataformas digitales.

Ante ello, Rita Segato (2016) reconoce la agencia bajo una concepción decolonial en "recuperar un tipo de politicidad cancelada a partir del secuestro de la enunciación política por la esfera pública, y la consecuente minorización [...] de la política de todos aquellos grupos de interés que no se ajusten a la imagen y semejanza del sujeto de la esfera pública" (p. 27). Segato plantea entonces la necesidad de otra manera de hacer política, una política femenina en espacios vinculares, de cercanía, bajo una noción de comunidad. La agencia se encuentra pues, en conformar una comunidad con densidad simbólica y una "autopercepción por parte de sus miembras de que vienen de una historia común, no desprovista de conflictos internos sino al contrario, y que se dirigen a un futuro en común" (Segato, 2016, p. 28).

En este sentido, la agencia se comprende en este artículo unificando las visiones de Wajcman (2006, p. 179) y Segato (2016, p. 28), como la capacidad del sujeto femenino para apropiarse de espacios digitales como arenas para generar una comunidad, comprendiendo que comparten una historia y un proyecto de dar continuidad a la existencia bajo un sujeto colectivo. Esto, en un entorno que, justo por su construcción sobre una cultura masculina de exclusión y dominación, las mujeres han demostrado resistencia al subvertir activamente la finalidad original de una determinada tecnología, en este caso, de las redes sociales.

\section{Hashtag feminismo: protesta feminista digital en México}


En este sentido, autoras como Esquivel (2019) y Pedraza y Rodríguez (2019) concuerdan que, en México, la emergencia de las movilizaciones feministas en redes sociales ha estado provocada por la violencia de género que las mujeres sufren en su cotidianeidad, donde sus experiencias compartidas en la red han estado discursivamente articuladas por medio de hashtags, "reafirmando su agencia para reivindicar su participación política" (Esquivel, 2019, p. 185).

A través del estudio cartografiado de los hashtags representativos de las protestas feministas en Twitter propuesto por Esquivel (2019), se recopilaron 47 hashtags surgidos a partir de un conflicto social de desigualdad o violencia contra mujeres y niñas, donde el hashtag haya marcado una tendencia nacional ( $\mathrm{y}$ en ocasiones internacional), y donde esta herramienta haya funcionado como catalizadora $\mathrm{u}$ organizadora de movilizaciones físicas. Entre los hashtags más comunes se encuentran \#NiUnaMas, \#NiUnaMenos, \#VivasNosQueremos, \#SiMeMatan, \#YoSiTeCreo, y el internacionalmente posicionado \#MeToo, del cual las usuarias mexicanas mostraron una apropiación al trasladarlo a sus contextos particulares, generando así una ola de hashtags como \#MeTooPeriodistasMexicanos, \#MeTooEscritoresMexicanos, \#MeTooArtesMX, entre otros.

La autora del estudio reconoció que, "al momento de hacer una denuncia por alguna forma de violencia, las usuarias hacen referencia a otros hashtags con algún tipo de similitud semántica" (Esquivel, 2019, p. 189), lo cual ha demostrado una relación constante de denuncias sobre tres grupos temáticos de relevancia para las mujeres en México: la violencia sexual y feminicida, la violencia sobre los derechos laborales de las mujeres y la violencia sobre sus derechos reproductivos. En palabras de Esquivel (2019), "en esta red de 47 hashtags es posible interpretar que las usuarias de Twitter continúan, no sólo generando nuevas formas de denunciar diferentes tipos de violencia, sino que hacen uso de hashtags que han marcado paradigmas discursivos y definen la agenda feminista en redes sociodigitales" (p. 193), lo que permite constatar que existe una dinámica de protesta con un sentido político determinado y evidente.

\section{Twitter y YouTube como espacios socio-técnicos}

\section{Twitter}

Lo aparición de esta protesta feminista a través de hashtags, responde al hecho de que Twitter supone un medio diseñado en un contexto tecnológico que se construye socialmente al mismo tiempo que permite la conglomeración de dinámicas sociales observables en un espacio mediado por tecnologías digitales, en gran parte debido a que está creado para provocar e incitar actualizaciones constantes en red por parte de sus usuarios entendidos como entes sociales (Murthy, 2012). Así, de acuerdo con Murthy (2012 p. 1061), esta plataforma presenta características puntuales de acceso gratuito, público, de multidifusión y conexión, donde la categoría de lo social adquiere significado en un diseño técnico que facilita la interacción, el intercambio y la colaboración.

Además, estos usos comunicativos que Twitter permite en su interfaz han demostrado una capacidad para moldear el acercamiento de los usuarios a la información y participación política (Maireder y Ausserhofer, 2014), en gran medida gracias a las capas estructurales que conforman de manera particular a esta red social. Ante ello, Axel Bruns y Hallvard Moe 
(2014) analizaron los nodos que componen a la plataforma estudiada, en la cual identifican tres niveles de comunicación fundamentales: el nivel micro de la comunicación interpersonal, el nivel meso de las redes de seguidores y el nivel macro de los intercambios basados en hashtags.

En primera instancia, en el nivel micro es posible observar la herramienta de "menciones", en la cual se utiliza el símbolo de la arroba (@) seguido del nombre del usuario con el cual se busca comenzar una conversación, o bien, a manera de respuesta frente a un tuit publicado por este mismo. Gracias a dicha función, cualquier usuario en Twitter puede ser aludido y convocado a alguna discusión particular, independientemente de si éste pertenece o no a la red de seguidores de quien lo menciona (Bruns y Moe, 2014, p. 19). Esto ha permitido que usuarios "comunes" tengan la capacidad de referenciar peticiones con tintes políticos a través de menciones a cuentas pertenecientes a figuras públicas o institucionales, que, si bien no necesariamente se obtiene una respuesta directa por parte de éstas, dicha práctica ha demostrado tener eficacia en la propagación colectiva de demanda ciudadana en tiempo real.

Ahora bien, el nivel meso en Twitter responde a la dinámica usuario/seguidores que, como mencionado anteriormente, a diferencia de otras redes sociales no requiere un seguimiento mutuo. Esta capa estructural supone entonces la naturaleza bilateral y pública que caracteriza a Twitter, donde los tuits publicados tienen la capacidad de llegar a todos los usuarios que siguen a la cuenta desde la cual fueron difundidos, pero que además son potencializados por la herramienta del retuit. A través del retuit, un tuit producido por un usuario tiene la posibilidad de ser compartido por más usuarios hacia sus particulares redes de seguidores, llegando así a nuevas y diversas audiencias más allá de las inmediatas y propias. Esto permite que los contenidos generados en Twitter transiten horizontalmente en el medio, respondiendo a la efectividad que el mismo tiene para la diseminación de mensajes noticiosos y demandas públicas (Bruns y Moe, 2014, p. 22).

Finalmente, la última capa estructural, concebida como el nivel macro de la plataforma, está constituida por formas y formatos que posibilitan una extensión aún mayor de alcance del contenido producido y compartido en esta red social. La principal herramienta de este nivel corresponde al hashtag —una palabra o concepto clave presidida por el símbolo del "hash" (\#) - la cual es comúnmente utilizada para marcar un tuit como relevante dentro de una discusión o tema en específico y hacerlo así más fácilmente descubrible para otros usuarios (Bruns y Moe, 2014, p. 17). Este mecanismo permite coordinar un intercambio de información relevante en temas de interés popular al posibilitar a los usuarios el rastreo de tuits que aparezcan bajo un hashtag en específico, independientemente de si el mensaje se originó desde una cuenta que ya seguían o desde una cuenta de un usuario previamente desconocido. De esta forma, incluir un hashtag en un tuit responde a un deseo de formar parte de un proceso comunicacional más extenso y activo, donde potencialmente se reúnan usuarios que, sin tener una conexión social previa, sostengan un mismo interés en un tema, noticia, evento o demanda particular a reportar.

De esta forma, el hashtag ha representado una herramienta de gran envergadura para el entendimiento sociotécnico de Twitter, al funcionar como un elemento de cohesión entre grupos y usuarios comunes que fuera de este plano digital posiblemente no encontrarían un espacio afín para abordar horizontalmente una conversación de interés colectivo. Así, las demandas y discursos políticos tienden a hacerse presentes en dicha plataforma, donde el 
fenómeno del hashtag permite agrupar y estructurar los debates y opiniones generadas frente a un tema o coyuntura específica (Bruns, 2012, en Maireder y Ausserhofer, 2014, p. 306).

\section{YouTube}

Por su parte, YouTube se ha presentado como un medio que permite democratizar la difusión de contenidos audiovisuales caseros en respuesta a un monopolio de producción de contenido por parte de cadenas televisivas y radiales, lo cual se ha reafirmado en su icónico slogan "Broadcast Yourself" (Valderrama y Velasco, 2018). Ante ello, algunos autores (O’Reilly, 2005, Campos Rodríguez, 2007) vieron en YouTube la posibilidad de generar contenido colaborativamente, permitiendo una amplia expresión de la identidad de los usuarios y posibilitando el desarrollo de comunidades libres del manejo de los medios tradicionales de comunicación. Sin embargo, de manera alterna a estas "benéficas capacidades de la plataforma”, como mencionado por Valderrama y Velasco (2018, p. 41), YouTube comenzó a ser utilizado por empresas y cadenas de medios convencionales para publicitar sus productos y ampliar las audiencias frente a sus contenidos. De esta forma, la plataforma demostró dinámicas de hibridez entre comercio y comunitarismo, y es considerada como un espacio "impulsado tanto por la industria como por los usuarios" (Snickars y Vonderau, 2009, p. 11).

A diferencia de las capas estructurales de comunicación discursiva en Twitter, YouTube basa su interfaz en la creación e intercambio de contenido multimedia. Justamente una de las características de esta plataforma es el reconocimiento o viralidad que pueden alcanzar los videos subidos a la misma. Cada video al momento de reproducirlo contiene a la derecha un listado de otros videos o contenidos relacionados, los cuales se dan gracias a que, al momento de cargar algún archivo multimedia, la plataforma solicita que el usuario le brinde un título, descripción y etiquetas; descriptores que son posteriormente utilizados por el sistema para agrupar semánticamente una lista de videos dentro de la misma temática (Siri, 2008). De igual forma, YouTube permite a los usuarios suscribirse a canales de su interés y marcar videos como favoritos, lo cual posibilita al diseño algorítmico de la plataforma generar patrones de atracción y posteriormente de recomendación para cada uno de los usuarios con base en su actividad dentro de la misma.

De acuerdo con Valderrama y Velasco (2018), estas características técnicas del espacio permiten identificar diferentes dinámicas de popularidad o viralidad que puede alcanzar un video compartido en YouTube. Una de ellas responde al reconocimiento social, alcanzado por un número determinado de visualizaciones y suscripciones donde los creadores de contenido toman en consideración las demandas y necesidades de su audiencia, privilegiando así el desarrollo y publicación de cierto tipo de contenidos sobre otros. Sin embargo, los autores reconocen un papel computable dado por la plataforma misma, donde sistemas automatizados de cuantificación y valorización digital "afectan las prácticas creativas al inscribir y hacer rastreables las interacciones (visualizaciones, likes, suscriptores, comentarios, etc.) de cada contenido. Tales interacciones, en términos agregados, configuran qué es popular en YouTube" (Valderrama y Velasco, 2018, p. 49), pero también se vuelven representaciones algorítmicas subjetivas del valor de cada canal y video compartido, por lo que "si bien teóricamente cualquiera puede lograr el "Broadcast Yourself", no todos los contenidos son igualmente visibles en la economía de la atención de YouTube” (Morreale, 2014, p. 118). 
En cuanto a los usuarios, YouTube cuenta con una cifra de 2,291 millones de usuarios activos (Shum, 2021) donde existen 37 millones de canales (Funk, 2020), por lo que solo un $1.6 \%$ del total de usuarios correspondería a creadores activos de contenido, donde el resto se limita únicamente a consumirlo y en ocasiones comentarlo. Por ende, aunque a diferencia de la televisión tradicional, en YouTube existe la posibilidad de interacción, participación y producción individual y colectiva, en términos y cifras prácticas el número de emisores o generadores de contenido es mucho menor al de receptores y consumidores. De acuerdo con José van Dijk, "esto pone de manifiesto que, por más que la plataforma haya buscado distinguirse inicialmente de otros medios, existe una fuerte heteronomía o imbricación entre la creación en YouTube, las marcas comerciales y los medios de comunicación tradicionales" (en Valderrama y Velasco, 2018, p. 49).

\section{Feminicidio de Monserrat Bendimes}

Para el análisis propuesto, se ha retomado el último caso mediático coyuntural en México, el cual corresponde al feminicidio de Monserrat Bendimes Roldán. Siguiendo en el presente artículo una mirada feminista, se retoma la necesidad que María Salguero (en Mendoza, 2020) expone de hacer hincapié en el registro de tantos detalles posibles sobre cada feminicidio incluyendo el nombre de la víctima, la edad, la relación con el perpetrador, el modo y el lugar de la muerte (D'Ignazio, y Klein, 2020, p. 143), como una forma de dar visibilidad a cada una de las víctimas y evitar su objetualización.

La siguiente información es retomada del reportaje realizado por Ángel Cortés Romero (2021) en el portal de La Silla Rota Veracruz (medio que se autodenomina como independiente), única fuente encontrada que recurría a la madre de Monserrat como fuente primaria de información, y seleccionada además por el cuidado tratamiento del encuadre empleado para dar forma a los datos presentados, además que pone en primer plano a Monserrat (o Monse) construyendo la historia alrededor de su persona, sus relaciones familiares y sus aspiraciones personales antes de su feminicidio.

Monse, de 20 años de edad, era una estudiante de Ingeniería Industrial en la Universidad del Valle de México, Campus Veracruz. Su familia, su madre, incluso su hermana - una de las piezas clave en el desarrollo de la conversación en Twitter sobre el feminicidio de Monse - la describen como una persona alegre y versátil, con sueños de continuar su preparación enfocándose en la nanotecnología y de completar sus estudios en una universidad de Alemania. En agosto del 2020 conoció a Marlon Botas Fuentes, uno de sus compañeros de carrera, quien se convirtió en su pareja y con quien sostuvo una relación de nueve meses de noviazgo.

El 17 de abril del 2021 Monse se encontraba en un bautizo familiar, para posteriormente ser acompañada por Marlon a su domicilio, aunque tenía planes de regresar a la celebración. Sin embargo, como relatado por su madre Cecilia Roldán, la familia recibió una llamada del padre de Marlon avisando que Monse estaba herida y se encontraba en el hospital, asegurándoles que no pasaba nada grave. No obstante, al llegar a la instancia, los padres de Monse descubrieron que su hija había sido víctima de un ataque a golpes por parte de Marlon Botas, quien le provocó fracturas en el cráneo, el cuello y brazos, y quien, tras dejarla en el hospital, huyó junto a su familia. 
Las lesiones infligidas por parte de Marlon causaron en Monse un estado crítico de salud, y de acuerdo con el relato de Cecilia, "fue Marlon" fueron las últimas palabras que Monse les compartió, antes de ser declarada con muerte cerebral por parte de los médicos que la atendían. Monse permaneció en terapia intensiva, pero tras seis días de hospitalización, su cuerpo dejó de responder ante las heridas perpetradas por el feminicida, Marlon Botas, ante lo cual fue desconectada y declarada muerta el 23 de abril del 2021.

\section{Metodología}

Ante el caso, en plataformas digitales pudo observarse generación de contenido, por un lado, en Twitter, donde surgió el hashtag \#justiciaparamonse, y en YouTube, donde fueron publicados videos correspondientes a spots noticiosos de medios como Milenio, Excélsior TV, Televisa, UnoTV, Imagen Noticias, etc., en los cuales usuarios y usuarias generaron comentarios sobre el caso expuesto.

Frente a ello, se llevó cabo una metodología de minería de datos digitales, recurriendo al scrapping de contenido a través de las API de cada una de las plataformas propuestas en el estudio. En el caso de Twitter, se realizó un scrapping en tiempo real del hashtag \#justiciaparamonse a través de la herramienta Workbench, la cual arrojó una base de datos de un total de 4,227 tuits generados bajo dicho hashtag entre las 11:08 p.m. del 23 de abril del 2021, y la 1:44 a.m. del 24 de abril del 2021, luego de darse a conocer la noticia del feminicidio de Monserrat.

Para obtener la data correspondiente de YouTube, se llevó a cabo el scrapping de los comentarios originados en dos videos encontrados en la plataforma. Se consideraron los videos de Milenio y UnoTv que fueron de igual forma arrojados por la red al momento de introducir la búsqueda bajo la frase "Justicia para Monse" y bajo el nombre de la víctima "Monserrat Bendimes", y seleccionados para el estudio con base en un mayor número de visualizaciones y comentarios generados con respecto al resto del contenido. El primero de ellos, compartido por el canal de Milenio, titulado "Muere joven que fue agredida por su novio en Veracruz; familiares exigen justicia", consta de 1:20 minutos de duración, 74,862 visualizaciones y un total de 305 comentarios por parte de usuarios; mientras que el segundo video elegido resultó perteneciente al canal de UnoTV, titulado "Justicia para Monse, exigen en redes: murió tras ser presuntamente golpeada por su novio" con 0:48 min de duración, 88,640 vistas y 114 comentarios reportados. Se realizó entonces un scrapping de los comentarios hallados en cada uno de los videos seleccionados a través de la herramienta Replit, obteniendo así un corpus total de 419 comentarios a analizar.

Puesto que el estudio se interesa en una indagación de las narrativas discursivas encontradas en Twitter y YouTube, se tomó como unidad de análisis el texto de ambas muestras, es decir, el contenido textual de los 4,227 tuits por una parte, y de los 419 comentarios en YouTube por otra. De esta forma, el contenido textual de cada uno de los corpus fue sometido a la plataforma Voyant Tools, herramienta web de lectura y análisis para textos digitales, con el fin de visualizar conceptos, correlaciones, frecuencias de términos y colocaciones encontradas en la muestra arrojada por cada plataforma a analizar.

\section{Resultados}


Al introducir a Voyant las bases de datos de Twitter y YouTube, se identificaron en primer lugar las siguientes visualizaciones de términos más utilizados en cada plataforma.

\section{Figura 1.}

Cirrus de corpus en YouTube

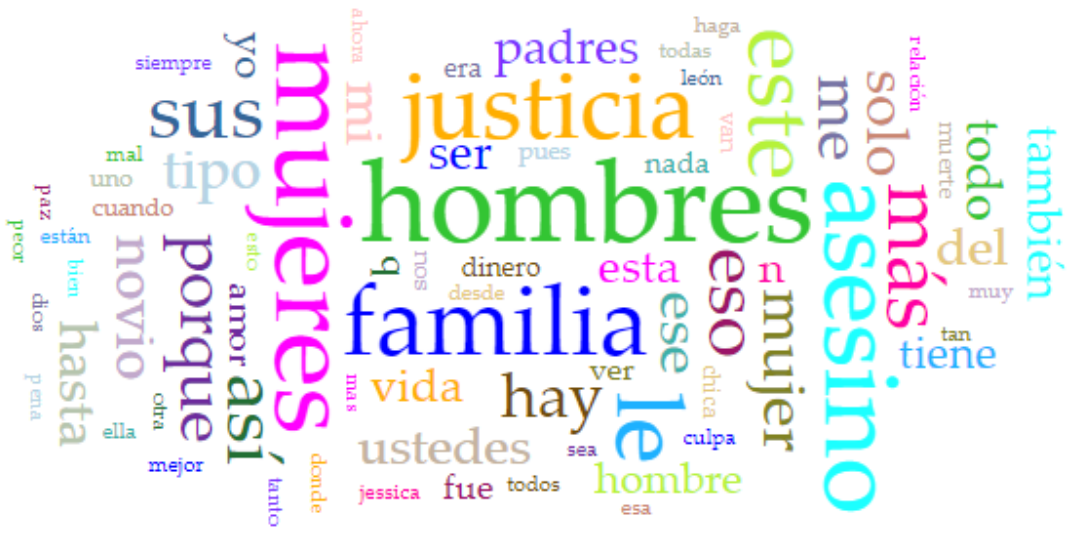

Por ende, en YouTube la palabra 'hombres' fue la más utilizada en los 418 comentarios analizados, seguido por 'mujeres' (50), 'familia' (46), 'asesino' (45), y finalmente 'justicia' (44) entre los más destacables.

En el caso de Twitter, el término más utilizado, naturalmente respondiendo a la naturaleza del hashtag, fue 'justiciaparamonse' apareciendo en 4,269 ocasiones dentro del corpus total, donde además destacan de manera fundamental para el análisis las palabras “monse” (1995), 'marlon' (1619) 'niunamenos' (1253), ‘justicia' (701), y el término 'feminicida' (615).

\section{Figura 2.}

Cirrus de corpus en Twitter

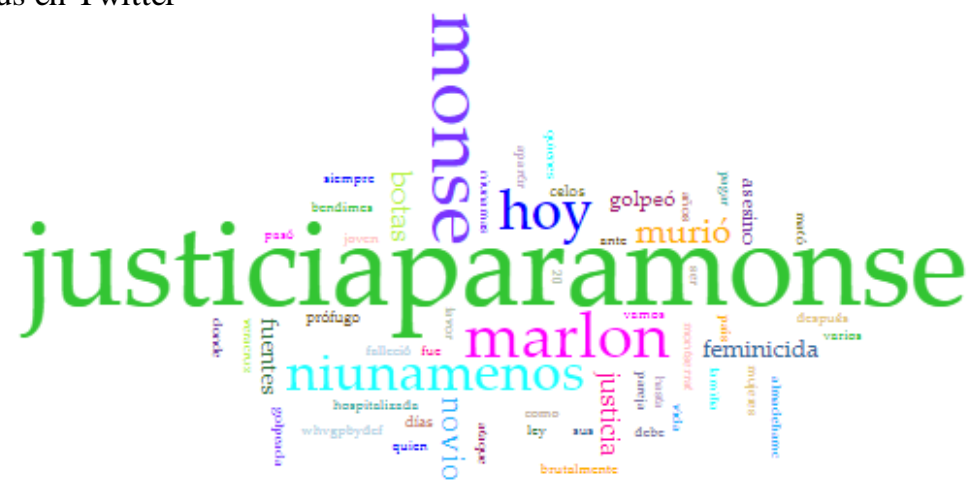
y 'murió':

En esta plataforma existe de igual manera una aparición continua de los términos 'no' 


\section{Figura 3.}

Términos de corpus en Twitter

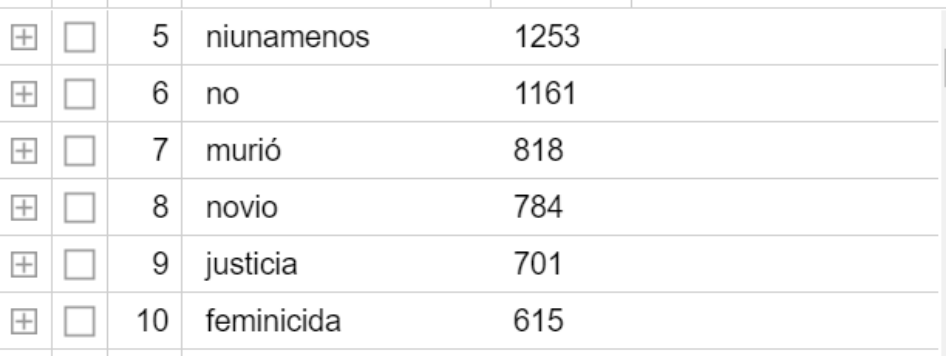

Que, cotejada con la figura 4 de colocaciones en el contexto, resulta de gran interés puesto que permite observar la introducción de una narrativa fundamental en la política discursiva feminista, la cual consiste en visibilizar un encuadre tradicionalmente hegemónico para el trato de un caso de feminicidio, en este caso el uso del término 'murió', que adjudica la muerte de manera pasiva a la víctima, para reconceptualizarlo a la acción directa perpetrada por el feminicida, donde se hace hincapié en el verbo 'mató'.

\section{Figura 4.}

Colocaciones de corpus en Twitter

\begin{tabular}{|l|l|l|}
\hline \multirow{2}{*}{$\begin{array}{l}\text { Cirrus } \\
\text { Término }\end{array}$} & Cómintexto del tér... & Contar (contexto) \\
\hline marlon & monse & 279 \\
\hline no & murió & 273 \\
\hline niunamenos & no & 269 \\
\hline no & niunamenos & 269 \\
\hline no & mató & 269 \\
\hline
\end{tabular}

\begin{tabular}{|c|c|c|c|}
\hline \multicolumn{2}{|r|}{ (1) Cirrus } & \multicolumn{2}{|c|}{ 柬 Colocaciones } \\
\hline & Término & Contexto del tér... & Contar (contexto) \\
\hline$\square$ & marlon & mató & 258 \\
\hline$\square$ & murió & marlon & 257 \\
\hline$\square$ & murió & justiciaparamonse & 254 \\
\hline$\square$ & justiciaparamonse & murió & 253 \\
\hline$\square$ & murió & mató & 252 \\
\hline
\end{tabular}

Regresando a la comparativa de corpus entre los comentarios encontrados en YouTube vs los tuits generados en Twitter, resulta de gran interés que la palabra 'justicia' aparece en ambos casos como una de las más utilizadas, por lo que puede deducirse en primera instancia que los públicos encontrados en ambas plataformas coinciden, en su mayoría, en la necesidad de exigir justicia y rendición de cuentas para Monse, la joven asesinada por su entonces pareja, Marlon Botas. Sin embargo, existen dos elementos importantes a resaltar en esta comparativa. La primera de ellas es que, en el caso de YouTube, no aparece, más que en seis ocasiones - como lo muestra la tabla 4- el nombre de la víctima de manera frecuente, mientras que, en Twitter, de acuerdo con la tabla 5, la palabra que corresponde al nombre de "monse" aparece en 1995 ocasiones dentro de los 4,227 tuits analizados, siendo el segundo término más frecuentado en el corpus mencionado.

\section{Figura 5.}

Términos de corpus en YouTube, palabra 'monse' 


\begin{tabular}{|c|c|c|c|c|c|}
\hline \multicolumn{3}{|c|}{ () Cirrus } & 目 Términos & \multicolumn{2}{|c|}{ ¿ Enlaces } \\
\hline & & & Términos & Contar & Tendencia \\
\hline 田 & $\square$ & 163 & méxico & 6 & \\
\hline$\boxplus$ & $\square$ & 164 & monse & 6 & \\
\hline 田 & $\square$ & 165 & muchos & 6 & \\
\hline$\boxplus$ & $\square$ & 166 & novios & 6 & \\
\hline
\end{tabular}

Figura 6.

Términos de corpus en Twitter, palabra 'monse'

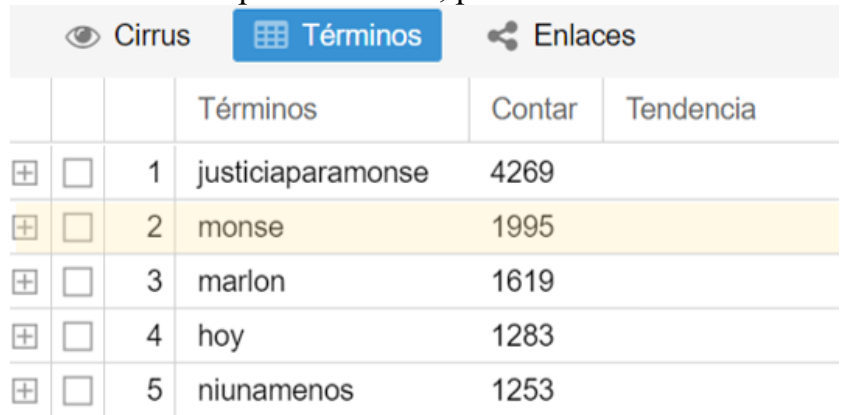

De lo anterior, puede explicarse que en Twitter existe un esfuerzo considerable por poner en el centro de la problemática a la víctima, además de referirla de manera constante directamente por su nombre, una práctica que se ha consolidado paulatinamente en el movimiento feminista. De acuerdo con María Salguero es necesario nombrar a las víctimas "por sensibilidad hacia ellas, para recordar que no son cifras, que cada víctima tiene un nombre" (en Mendoza, 2020). Por el contrario, en YouTube los usuarios no resaltan la necesidad de enfatizar el nombre de la víctima en sus comentarios, por lo que escapan a esta dinámica conectiva que sí es observada en Twitter y que permite suponer la existencia en esta última plataforma de un entendimiento colectivo más notorio de los elementos presentes en la lucha feminista frente al fenómeno.

En segundo lugar, se resalta que, en el corpus analizado de YouTube, aparece la palabra 'asesino' como cuarto término más utilizado por los usuarios al momento de generar comentarios en los videos que exponen el caso estudiado.

\section{Figura 7.}

Términos de corpus en YouTube, palabra 'asesino' 


\begin{tabular}{|c|c|c|c|c|c|}
\hline \multicolumn{3}{|c|}{ (1) Cirrus } & 雷 Términos & \multicolumn{2}{|c|}{$\propto$ Enlaces } \\
\hline & & & Términos & Contar & Tendencia \\
\hline$\boxplus$ & $\square$ & 1 & hombres & 51 & \\
\hline$\boxplus$ & $\square$ & 2 & mujeres & 50 & \\
\hline 田 & $\square$ & 3 & familia & 46 & \\
\hline 田 & $\square$ & 4 & asesino & 45 & \\
\hline$\boxplus$ & $\square$ & 5 & justicia & 44 & \\
\hline
\end{tabular}

Por otro lado, en Twitter se observa el uso de la palabra 'feminicida', encontrándose en la posición número 10 (tabla 7) y representando una alta frecuencia de aparición en 615 tuits del corpus analizado, mientras que, en los comentarios de YouTube, este término sólo aparece en 5 ocasiones, ocupando el lugar número 194 del corpus (tabla 8).

\section{Figura 8.}

Términos de corpus en Twitter, palabra 'feminicida'

\begin{tabular}{|c|c|c|c|c|c|}
\hline \multicolumn{3}{|c|}{ (1) Cirrus } & 柬 Términos & \multicolumn{2}{|c|}{ Enlaces } \\
\hline 4 & 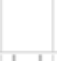 & z & Términos & Contar & Tendencia \\
\hline 田 & $\square$ & 6 & no & 1161 & \\
\hline 田 & $\square$ & 7 & murió & 818 & \\
\hline 田 & $\square$ & 8 & novio & 784 & \\
\hline 田 & $\square$ & 9 & justicia & 701 & \\
\hline 田 & $\square$ & 10 & feminicida & 615 & \\
\hline
\end{tabular}

\section{Figura 9.}

Términos de corpus en YouTube, palabra 'feminicida'

\begin{tabular}{|c|c|c|c|c|c|}
\hline \multicolumn{3}{|c|}{ () Cirrus } & 浑 Términos & \multicolumn{2}{|c|}{$<$ Enlaces } \\
\hline & & & Términos & Contar & Tendencia \\
\hline$\boxplus$ & $\square$ & 193 & favor & 5 & \\
\hline$\boxplus$ & $\square$ & 194 & feminicida & 5 & \\
\hline 田 & $\square$ & 195 & feminicidio & 5 & \\
\hline 田 & $\square$ & 196 & fuera & 5 & \\
\hline 田 & $\square$ & 197 & gracias & 5 & \\
\hline
\end{tabular}

La variabilidad de frecuencias observadas para el término 'feminicida' en ambos corpus resulta de alta relevancia para la investigación, puesto que, aunque en YouTube se 
identifica, en este caso, a Marlon Botas como 'asesino', los usuarios en esta plataforma escapan de la narrativa observada mayormente en Twitter que tipifica como 'feminicida' al perpetuador del crimen. De acuerdo con la teoría feminista, la correcta tipificación del fenómeno resulta fundamental puesto que "además de visibilizar la forma extrema de violencia contra las mujeres, tiene como fin garantizar un seguimiento adecuado y especializado que pueda prevenir, atender, sancionar y erradicar esta problemática" (Araiza Díaz et al., 2020).

Por ello, mientras que en Twitter — según los datos arrojados en el análisis- existe una tendencia mayor a identificar y tipificar correctamente los elementos presentes en el fenómeno del feminicidio, en YouTube puede observarse que los usuarios responden a lo que Rita Segato (2006) denomina como "voluntad de indistinción", donde se "estimula una percepción indiscriminada de la cantidad de crímenes misóginos" (p. 16), como una resistencia para generar tipologías precisas en la materia, dificultando así abordar de manera precisa la problemática desde una perspectiva estructural de violencia de género y no como un caso aislado de asesinato.

\section{Revictimización en YouTube}

Como anteriormente mencionado, la voluntad de indistinción junto con la incapacidad de tipificar y nombrar de manera precisa los elementos que juegan un rol importante en el entendimiento del feminicidio como una problemática sistémica, pueden devenir en la mitificación del fenómeno, y como consecuencia, en un entendimiento normativo social del mismo. Teniendo en cuenta que la tradición de los medios convencionales mexicanos ha sido partícipe de esta nombrada voluntad de indistinción, y considerando lo hasta ahora analizado y arrojado por el corpus encontrado en YouTube, la investigación se interesó por identificar la cantidad de comentarios que, más allá de escapar a la terminología correcta del fenómeno, responden además a una metanarrativa de encuadre hegemónico donde se culpabiliza a la víctima.

Para ello, se analizaron los 418 comentarios del corpus total de YouTube, y se extrajo que, en un 19\% de ellos, es decir, en 79 comentarios, los usuarios recurrieron a narrativas de revictimización, cuestionamiento a la víctima o al género femenino y culpabilización directa. Se creó una nueva base de datos únicamente con estos comentarios, la cual fue sometida a análisis en la herramienta Voyant, obteniendo la siguiente gráfica de visualización de términos más utilizados.

\section{Figura 10.}

Cirrus de corpus en YouTube - Revictimización

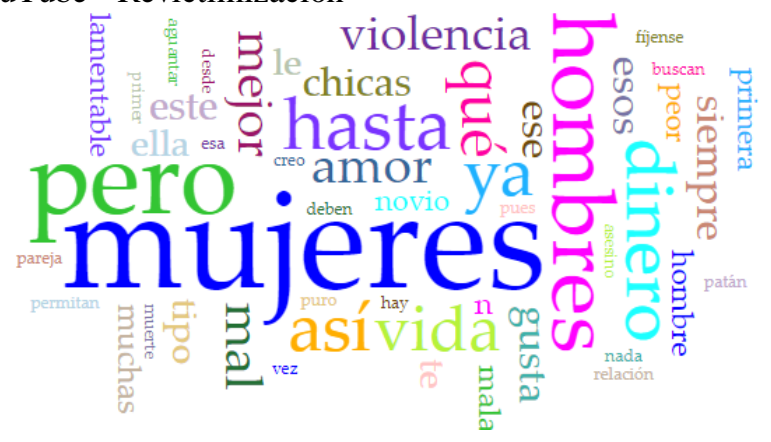


Destacan en ella las palabras 'mujeres' con 24 frecuencias, 'pero' con 16, 'hombres' apareciendo en 12 ocasiones, así como 'dinero' con 10 usos dentro del corpus seleccionado.

Una de las gráficas más interesantes arrojadas por la herramienta, corresponde a la de 'Enlaces', la cual permite observar las relaciones entre palabras más utilizadas, presentada en la gráfica a continuación:

\section{Figura 11.}

Enlaces de corpus en YouTube - Revictimización

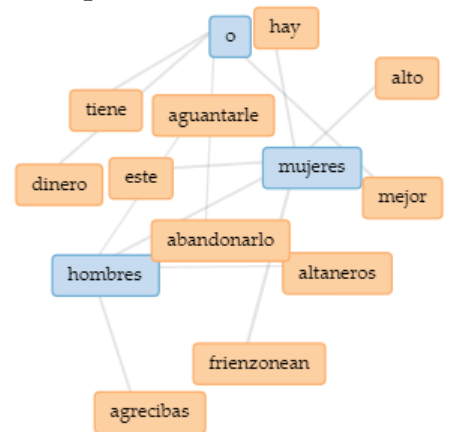

En ella es posible detectar un discurso donde la mayoría de los comentarios responsabiliza a la víctima, extendiendo la narrativa a "todas las mujeres" con comentarios como:

\section{Figura 12.}

Comentarios recopilados en YouTube de revictimización

Es lamentable pobre chica pero las que deben cambiar son ellas que les encantan los chicos malos se acuerdan del mata novias y así cientos de weyes así

Que mal que prefirió morir, antes que denunciar a ese sujeto, no creo que sea la primera vez que la golpeaba, lo malo es que hay muchas mujeres pasando por lo mismo en este momento, y de igual forma, prefieren perdonarle los maltratos a el patán en turno, antes de abandonarlo, solo porque, o tiene dinero, es guapo, o porque le pidio perdon y dijo que es la ultima vez.

Otra que sabiendo que son violentos siguen ahí... Haaaa pero en redes ponen me trajo rosas

De igual manera, se culpabiliza a la víctima de su propio feminicidio, en muchas ocasiones ligándolo a cuestiones de conveniencia por dinero ${ }^{1}$, ambición ${ }^{2} \mathrm{o}$ infidelidad. ${ }^{3}$

\section{Figura 13.}

Comentarios de ejemplificación 


\section{Ella fue la culpable por ambiciosa del dinero \\ 2 Todo por avara y ambiciosa \\ 3 Eso le pasa por infiel}

De acuerdo con Rita Segato (2016), estas atribuciones responden a "una explicación reduccionista y simplificadora por ser monocausal, $[\ldots]$ por tratarse de una explicación referida a emociones privadas, a los afectos de fuero íntimo, cuando en realidad estamos frente a un panorama configurado por intereses de órdenes que superan en mucho la esfera de la intimidad" (pp. 81-82). A pesar de que los videos per se de los cuales se extrajo el corpus analizado presentan la noticia de forma neutral, los comentarios tendieron en un $19 \%$ a recurrir a narrativas revictimizadoras y simplificantes del feminicidio, de lo cual puede deducirse que, como anteriormente referido, existe aún una notable normalización social arraigada en el imaginario colectivo frente al fenómeno.

\section{Sujeto de la enunciación en Twitter}

Teniendo en cuenta esta importante diferenciación de narrativas entre plataformas, y reconociendo en Twitter, a diferencia de YouTube, un espacio más amplio para el posicionamiento de resistencia a las meta-narrativas que convencionalmente se han hecho presentes en los casos de feminicidio en México, resulta pertinente reconocer quiénes son los o las sujetos de enunciación que participan de la conversación en esta red social. Frente a ello, se realizó a través del corpus arrojado por Workbench un análisis del engagement presentado en los tuits recuperados para tener un acercamiento a los usuarios/usuarias con mayor relevancia en la discusión digital del feminicidio de Monserrat Bendimes.

De esta manera, se recuperaron los 15 tuits con mayor engagement (dado por la cantidad de retuits y favoritos recibidos) presentado al momento de la extracción de la muestra, recuperando las cuentas de usuarios y usuarias que los generaron y sometiéndolos a un análisis de actividad en la plataforma a través de Botometer para descartar una naturaleza artificial como bots. Ante ello, se recopilaron las siguientes cuentas:

\section{Figura 14.}

Resultados de Botometer de las 12 cuentas con mayor número de engagement en la muestra analizada. Las puntuaciones bajas indican cuentas humanas probables y puntajes más altos significan más actividad similar a un bot. 


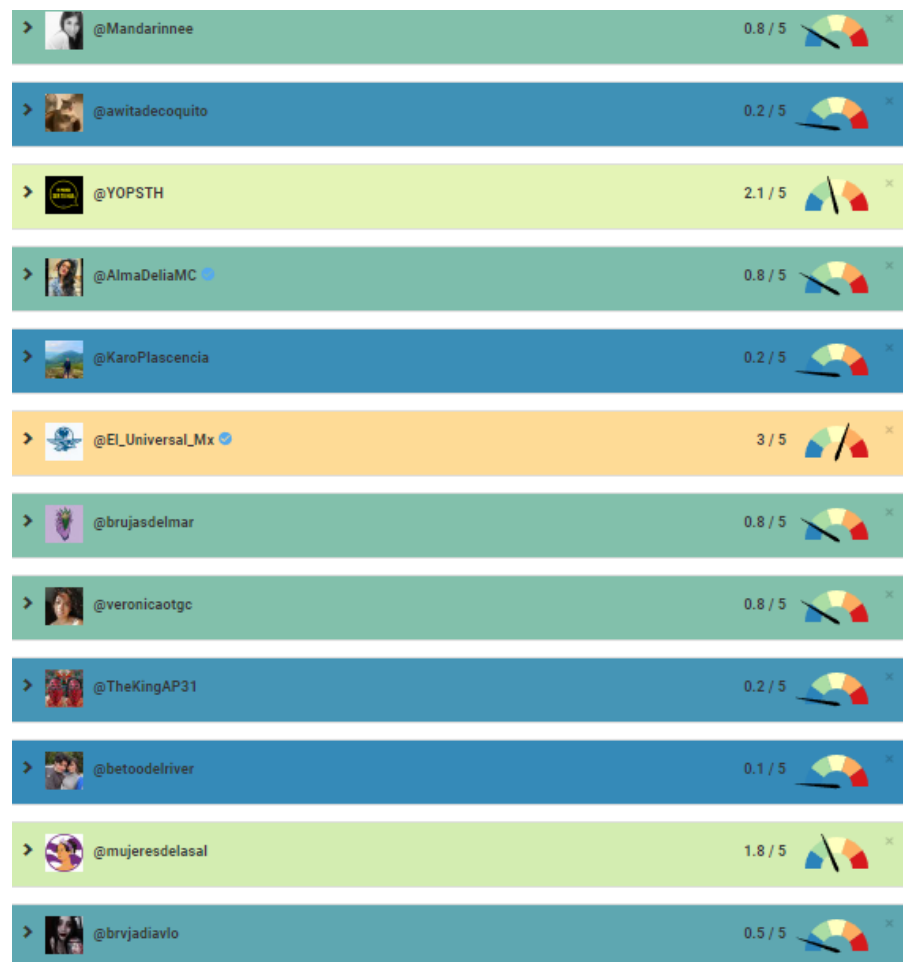

En un análisis de lectura cercana a las cuentas recuperadas (análisis de comportamiento en la plataforma a través de Botometer y observación directa de los perfiles en Twitter), fue posible observar que 6 de los tuits con mayor engagement pertenecen a cuentas de colectivas feministas mexicanas, entre ellos Las Brujas del Mar, Mujeres de la Sal y Yo Podría Ser tu Hija Puebla (@YOPSTH). En segundo lugar, se observó que 5 de los tuits más viralizados corresponden a cuentas ordinarias de usuarias mujeres que propiciaron la conversación en el medio (@mandarinnee, @awitadecoquito, @KaroPlascencia, @ veronicaotgc, @brvjadiavlo) además de una cuenta verificada correspondiente a Alma Delia Murillo, escritora mexicana. De igual manera se rescata que aparecen en la conversación dos tuits generados por usuarios hombres y un tuit de corte periodístico por parte del periódico $E l$ Universal.

Esto constata que, si bien existen diversas voces en la conversación al ser Twitter una plataforma de acceso abierto existe en efecto una apropiación importante por parte de colectivas feministas y usuarias mujeres en la discusión del caso y del fenómeno en Twitter. En el caso de las colectivas, Natalia Aruguete (2021, p. 2) sostiene que para que se conformen narrativas en red en una plataforma como lo es Twitter, es necesario que las y los usuarios activen mensajes que les son ofrecidos y no impuestos, muchas veces provenientes de autoridades en el tema o fenómeno debatido dentro de la red social. Por autoridades se entiende en este caso a los autores o autoras de los tuits originales que propagan información a más velocidad y con mayor alcance debido a algún tipo de nivel jerárquico dentro de la 
plataforma, ayudados además por la influencia de sus narrativas (Aruguete, 2020, p. 79). Lo anterior se ha visto ya antes reflejado en dinámicas de hashtag feminismo, como por ejemplo en el caso del hashtag \#VivasNosQueremos, el cual fue impulsado en el 2014 por la colectiva feminista mexicana Mujeres Grabando Resistencias (Camusso y Rovetto, 2014, p. 163), donde se ha demostrado, como en este caso, que las colectivas han tenido un peso importante en "procesos de apropiación tecnológica de las mujeres [...] para dar paso a prácticas de organización, expresión, cuestionamiento y resistencia” (Pedraza y Rodríguez, 2019, p. 210).

En el caso de las usuarias, y aunque en la muestra analizada no se observa directamente esta presencia, vale la pena rescatar el papel que la hermana de Monse, Leslie Bendimes, ha tenido en la visibilidad del caso en Twitter. La ausencia de su usuario (@LeslieBr8) en la muestra de este estudio puede deberse a que la misma fue recogida en un lapso inmediato al anuncio de la defunción de Monse; sin embargo, su voz se ha convertido de igual manera en una voz dominante que activa y reactiva constantemente la memoria del feminicidio de su hermana en la plataforma. 


\section{virtunglis}

\section{Figura 15.}

Recopilación de tuits extraídos de [@LeslieBr8]
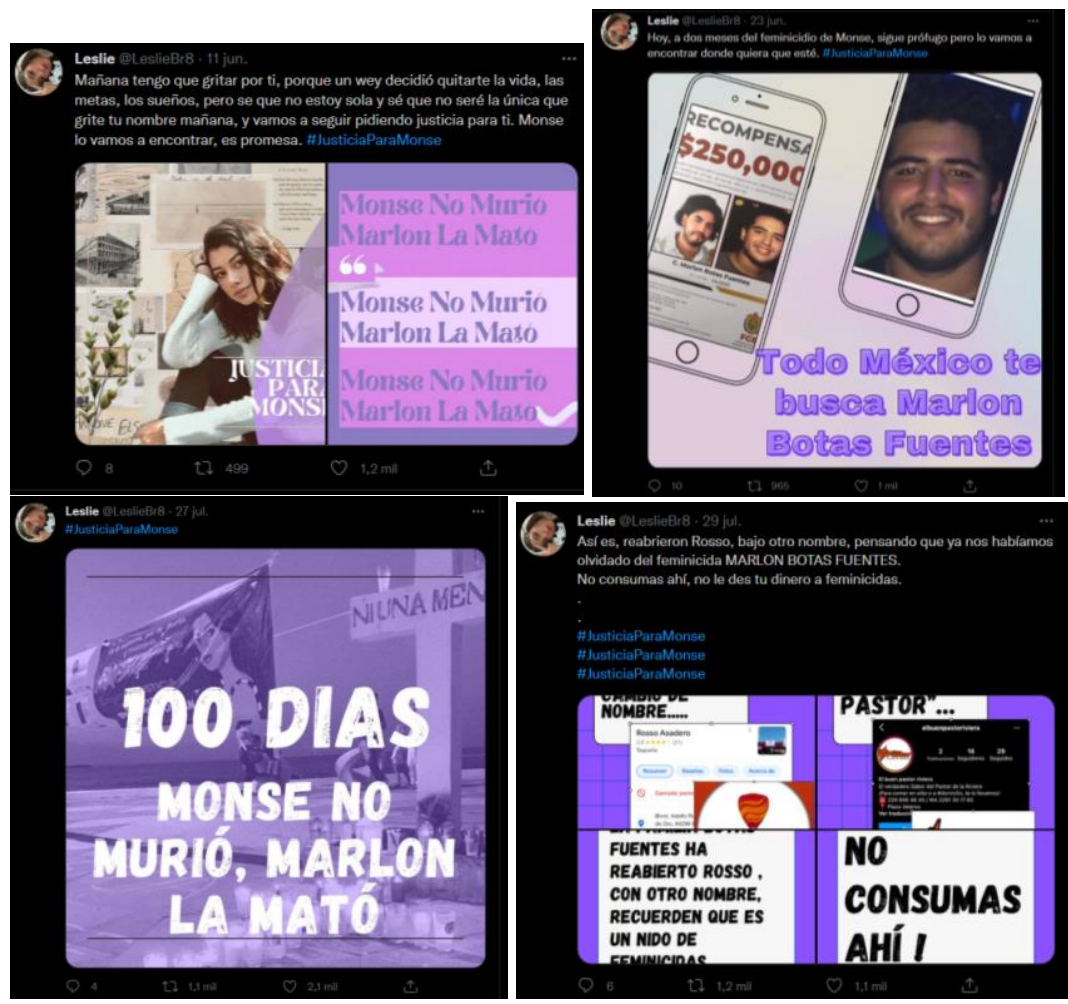

Observando la tipificación a la que recurre Leslie constantemente del caso de su hermana como feminicidio y siguiendo una narrativa apegada a la visibilización feminista del fenómeno, se materializa concretamente la noción que Wajcman (2006) sostiene sobre la factibilidad de identificar al sujeto femenino dentro de estas redes tecnológicas. Esto en un esfuerzo por posicionar no solo el caso en la discusión pública, sino también los términos, narrativas y un tratamiento que recupera a la víctima, que la nombra y cuya justicia se busca no sólo en la rendición de cuentas por parte del feminicida, sino también en mantener la memoria como un motor de la lucha feminista en un ímpetu por incidir en las convenciones sociales existentes alrededor del fenómeno.

Por ende, es posible reconocer en el papel de Leslie, así como en las usuarias que forman parte de la discusión y visibilización del caso, la agencia que las mujeres sostienen en la subversión de construcciones convencionales de pasividad en un espacio como lo es Twitter, algo que Segato (2016) reconocería como agencia al remontar "el hilo de la memoria y los fragmentos de tecnologías de sociabilidad que están entre nosotras hasta recuperar el tiempo en que [...] las formas de contacto interpersonal no habían sido desplazadas y clausuradas por la emergencia de la esfera pública de genealogía masculina” (p. 31). 


\section{Conclusiones}

Analizando las herramientas sociotécnicas propuestas, en este caso YouTube y Twitter, fue posible deducir, en primer lugar, que YouTube demostró —al menos en el caso particular estudiado - no responder realmente a un espacio alterno para el surgimiento de narrativas distantes al tratamiento tradicional que los medios convencionales en el país han dado en su mayoría al fenómeno y a los casos de feminicidio. Lo anterior, posiblemente atribuible al contexto de producción de los videos de los cuales se extrajo la muestra a analizar, puesto que únicamente se encontró contenido audiovisual en la plataforma que respondía a spots de noticias pertenecientes a la prensa convencional mexicana, y no a videos creados por usuarios o usuarias donde pudiese existir un discurso de contranarrativa o, incluso, activismo frente al tema.

Aunque existieron en YouTube narrativas de exigencia de justicia, lo cierto es que, frente a una voluntad de indistinción plagada de concepciones aún misóginas del fenómeno, Segato (2016) argumenta que "los estereotipos afectan negativamente las prácticas de investigación policial y jurídicas, así como la administración de una justicia capaz de contemplar la queja de las víctimas" (p. 87). Por ello, en esta plataforma se pueden observar reproducciones de poder basadas en nociones patriarcales y machistas del fenómeno del feminicidio, mismo que puede explicarse, de acuerdo con Castillo González (2018, p. 115), al hecho de que esta plataforma tiene funciones y usos que la sitúan como una propuesta posttelevisiva de la modernidad tardía, aunado a la implicación automatizada de los algoritmos que posicionan más bien a YouTube como un espacio donde tienden a reproducirse discursos infundados en concepciones patriarcales del feminicidio.

Esto es lo que Wajcman (2006, p. 86) advierte en la visión ultra optimista del ciberespacio como una zona sin límites para las mujeres que escape de un control patriarcal, pues, aunque estas arenas presentan nuevas posibilidades para la presencia de las agendas feministas, el caso analizado en YouTube demuestra que existen en la plataformización continuidades de dominación y exclusión masculinas, aunque lo hagan bajo formas nuevas.

Sin embargo, cosechar una postura pesimista del espacio tecnológico únicamente con base en dicho descubrimiento, excluiría la propuesta tecnofeminista de esta autora de reconocer el carácter sociotécnico y maleable de la tecnología, y en él, dinámicas importantes de resistencia a dichas continuidades de dominación observadas en redes digitales. Esto se constata en el caso de Twitter donde, gracias al diseño técnico de la plataforma, incluso la generación misma del hashtag estudiado bajo la palabra 'justicia' logra poner en el centro de la problemática y del caso de feminicidio a la víctima, en contraposición al parámetro hegemónico encontrado en la prensa tradicional en la cual existe una tendencia mayor a justificar al victimario (Tiscareño y Miranda-Villanueva, 2020).

De esta manera, la creación, movilización y visibilidad que adquiere el hashtag para introducir una narrativa en red de contranarrativa al tratamiento tradicional del fenómeno, materializa una mayor capacidad y dinámica de agencia y política discursiva feminista que ha permitido a las mujeres, a través de colectivas, voces dominantes (como en este caso, la de Leslie Bendimes) y, en general, de usuarias, apropiarse con mayor ímpetu de este espacio para ampliar la discusión pública de estos acontecimientos y, en general, del fenómeno del feminicidio en el país. 
Recuperando esta visión, el presente artículo reconoce la agencia en un contexto decolonial conjuntando las propuestas de Wajcman (2006) y Segato (2016) como una capacidad de apropiación del espacio digital por parte del sujeto femenino que, frente a un fenómeno de alta sensibilidad y urgencia como lo es el feminicidio, introduce narrativas en red que generan resistencia a una esfera pública donde los medios convencionales han minimizado y mitificado la problemática estructural de violencia de género vivida en un país como México, arraigando imaginarios colectivos de normalización de la misma. Así, en Twitter a través de un hashtag como \#justiciaparamonse, las mujeres y los colectivos feministas han posicionado en el debate público la necesidad de tipificar correctamente, de nombrar a la víctima, de visibilizar a la problemática como estructural, reconociendo una política femenina de los vínculos como Segato (2016, p. 27) defiende. Esto materializa la propuesta de Wajcman (2006) de reconocer que el sujeto femenino no sólo puede, sino que debe valerse del carácter sociotécnico de la tecnología, en este caso, de un espacio digital, para evitar que en él se reproduzcan sin fronteras las dinámicas constitutivas del género, y así subvertirlas activamente al apropiarse de estas arenas con sus propias dinámicas, agendas y en este caso, narrativas en red.

\section{Referencias}

Araiza Díaz, A., Vargas Martínez, F. C., y Medécigo Daniel, U. (2020). La tipificación del feminicidio en México. Un diálogo entre argumentos sociológicos y jurídicos. Revista interdisciplinaria de estudios de género de El Colegio de México, 6, 468.

Aruguete, N. (2021). Activación de encuadres en red. Un modelo para repensar la circulación de sentidos en el nuevo entorno mediático. Profesional de la información, 30 (2), 118.

Aruguete, N. (2020). Activación de encuadres mediáticos en redes sociales. En E.Actis, M. Berdondini y S. Castro Rojas (Comps.), Ciencias Sociales y Big Data (pp. 65-83). UNR Editora.

Bruns, A., y Moe, H. (2014). Structural layers of communication in Twitter. En K. Weller, A. Bruns, J. Burgess, M. Mahrt y C. Puschmann (Eds.), Twitter and Society (pp. 15-28). Peter Lang Media.

Campos Rodríguez, J. (2007). ¡Broadcast Yourself! Identidad, comunidad y masas inteligentes para la nueva sociedad del conocimiento. ICONO14 Revista Científica de Comunicación y Tecnologías Emergentes, 5(1), 160-192.

Camusso, M., y Rovetto, F. (2016). \#Ni una (imagen) menos. Imágenes, apropiaciones y circulación en las redes sociales. Nuevas mediatizaciones: nuevos públicos, pp. 159178. 
Castillo González, M. (2018). Narrativas de reconocimiento y justicia en Red. El videoactivismo por Ayotzinapa en YouTube. [Tesis doctoral, Tecnológico de Monterrey]. https://bit.ly/3I4KkpA

Cortés Romero, A. (2021, 25 de octubre). "Marlon nos dio una puñalada"; feminicidio de Monse, 6 meses sin justicia. La Silla Rota Veracruz. https://bit.ly/3pb1VTY.

D’Ignazio, C., y Klein, L. (2020). Data Feminism. The MIT Press Open.

Esquivel, D. (2019). Construcción de la protesta feminista en hashtags: aproximaciones desde el análisis de redes sociales. Comunicación y medios, 28(40), pp. 184-198.

Forbes Staff. (2020, 10 de noviembre). Sólo 1 de cada 2 feminicidios son castigados en México. Forbes. https://bit.ly/3xpN37X.

Funk, M. (2020, 13 de noviembre). How many YouTube channels are there? Tubics. https://bit.ly/3DVujQj.

Guillén. B. (2021, 28 de junio). Los feminicidios en México aumentan un 7,1\% en los cinco primeros meses de 2021. El País. https://bit.ly/3nOHCfI.

Hernández Castillo, R. A. (2003), Posmodernismos y feminismos: diálogos, coincidencias y resistencias. Desacatos, 13, 107-121.

Hernández Montoya, E. (2020). ¿Cómo se representan los feminicidios en los medios de comunicación? Catedra UNESCO-AMIDI. https://bit.ly/3nReUuw.

Maireder, A., y Ausserhofer, J. (2014). Political Discourses on Twitter. En K. Weller, A. Bruns, J. Burgess, M. Mahrt y C. Puschmann (Eds.), Twitter and Society (pp. 305318). Peter Lang Media.

McNeill, S. (2006). Matón de mujeres convertido en héroe trágico. En Russell, D. y Radford, J. (Eds.), Feminicidio: la política del asesinato de las mujeres. UNAM.

Mendoza, V. (2020, 16 de junio). Mujeres Poderosas 2020: María Salguero ubica y pone nombre a víctimas de feminicidio. Forbes. https://bit.1y/3nSf059

Montanaro, A. (2017). Una mirada al feminismo decolonial en América Latina. Universidad Carlos III de Madrid.

Morreale, J. (2014). From homemade to store bought: Annoying orange and the professionalization of YouTube. Journal of consumer culture, 14(1), 113-128.

Murthy, D. (2012). Towards a sociological understanding of social media: Theorizing Twitter. Sociology, 46(6), 1059-1073.

Natansohn, G., y Paz, M. (2018). “Ciberfeminismos contemporáneos, perspectivas desde América Latina”. $8^{\circ}$ Conferencia Latinoamericana y Caribeña de Ciencias Sociales.

O'Reilly, T. (2005, 30 de septiembre). What is Web 2.0: Design patterns and business models for the next generation of software. O'Reilly. https://bit.ly/3oUSXtO.

Page, R., Harper, R. H. R., y Frobenius, M. (2013). From small stories to networked narrative: The evolution of personal narratives in Facebook status updates. Narrative Inquiry, 23(1), 192-214.

Papacharissi, Z. (2014). Affective publics: Sentiment, technology, and politics. Oxford University Press.

Pedraza, C., y Rodríguez, C. (2019). Resistencias sumergidas. Cartografía de la tecnopolítica feminista en México. Teknokultura, 16(2), pp. 197-212.

Reyes-Rivas, R. (2017). Cyborgs en Centroamérica: ciberespacio, políticas oposicionales y pensamiento feminista decolonial. Interritórios, 3(4), 153-164. 
Romero, A. (2014). La utopía postfeminista. Del ciberfeminismo al tecnofeminismo. Cuadernos del Ateneo, 156-169.

Sandoval, C. (2010). De los medios a los fines. La comunicación como bien público. Revista Latinoamericana de Comunicación, 13, 60-70.

Segato, R. (2006). ¿Qué es un feminicidio? Notas para un debate emergente. Serie Antropología, 401, 2-11.

Segato, R. (2016). La guerra contra las mujeres. Traficantes de Sueños. Madrid.

Shum, Y. (2021, 9 de febrero). Resumen de YouTube 2021: 2.291 millones de usuarios activos. Yi Min Shum Xie. https://bit.ly/3xmCbrD.

Siri, L. (2008). Un análisis de YouTube como artefacto sociotécnico. Diálogos de la Comunicación, 77(9), s.p.

Snickars, P., y Vonderau, P. (2009). Introduction. En P. Vonderau y P. Snickars (Eds.), The YouTube reader (pp. 9-21). Stockholm: National Library of Sweden.

Tiscareño, E., y Miranda-Villanueva, O. (2020). Víctimas y victimarios de feminicidio en el lenguaje de la prensa escrita mexicana. Comunicar: Revista Científica de Educomunicación, 63(28), 51-60.

Valderrama, M. y Velasco, P. (2018). ¿Programando la creación? Una exploración al campo sociotécnico de YouTube en Chile. Cuadernos.info, 42, 39-53.

Wajcman, J. (2006). Tecnofeminismo. Cátedra. 